\title{
Electrocutaneous stimulation II. The estimation of distance between two points
}

\author{
LAWRENCE E. MARKS \\ John B. Pierce Foundation Laboratory and Yale University, New Haven, Connecticut \\ JOHN P. GIRVIN \\ The University of Western Ontario, London, Ontario, Canada \\ and \\ DONALD O. QUEST, JOHN L. ANTUNES, PETER NING, \\ MICHAEL D. O'KEEFE, and Wm. H. DOBELLE \\ College of Physicians and Surgeons of Columbia University, New York, New York
}

\begin{abstract}
Using a numerical estimation procedure, subjects judged the perceived distance between pairs of brief electrical stimuli presented over different physical distances, on different body regions, and in different spatial orientations. The skin turns out to be anisotropic, with the same physical separation between electrodes appearing greater on the forehead than on the forearm, greater on the forearm than on the abdomen. Perceived distance also depende on the orientation and, to some extent, on the time relation between the two electrical pulses. Under all conditions, the perceived distance between electrical stimuli was "compressed" as compared with visual distance. These results bear on the design of devices that attempt to transmit information by electrical stimulation of the skin.
\end{abstract}

Having evaluated, in the previous paper of this series (Girvin, Marks, Antunes, Quest, O'Keefe, Ning, \& Dobelle, 1982), how several properties of the stimulus influence absolute sensitivity to electrocutaneous stimulation, the specific aim of the experiments reported here is to determine the skin's capacity to represent spatial information. In essence, this study asks: How accurately and how reliably can one perceive the distance between two brief electrical pulses presented in various parts of the body?

It has long been known that the skin is not isotropic in its response to mechanical stimulation. More than a century ago, E. H. Weber (1834) observed that the distance between two points touched on the skin seemed greater in some parts of the body (e.g., the face) than in others (e.g., the trunk), and greater when the points were oriented in certain directions (e.g., transversely) than in others (longitudinally). In terms of the two-point threshold, measurements by others (Vierordt, 1869; Weinstein, 1968) conform to Weber's own observations, although, to be sure, two-point thresholds provide

This research was supported by a grant from the National Science Foundation (PFR 79-17634). Reprints may be requested from Lawrence E. Marks, John B. Pierce Foundation Laboratory, 290 Congress Avenue, New Haven, Connecticut 06519. D. Quest and J. L. Antunes are affiliated with the Neurological Institute of New York, and P. Ning, M. D. O'Keefe, and W. H. Dobelle are affiliated with the Division of Artificial Organs, both of the College of Physicians and Surgeons of Columbia University. an indirect measure of perceived distance (it is necessary to make the assumption that when the twopoint threshold is smaller, a given physical distance will contain more threshold units and therefore will yield a greater perceived distance than when the twopoint threshold is larger).

A more recent and more direct attack on this question is that of Green (1982), who asked subjects to make magnitude estimates of the perceived distance between two mechanical pulses presented at different body sites and in different orientations. Green found and quantified the skin's anisotropy in that, for example, the same physical distance appeared greater when presented transversely on the forearm than when presented longitudinally. In virtually all body regions, Green found tactual distance to be "compressed" as compared with visual distance-that is, the same physical distance appeared greater to the eye than to the skin.

Less is known about the skin's ability to represent spatial information presented electrically, although there have been a few measurements of the electrocutaneous two-point threshold. Jones (1956) found two-point thresholds to decrease with increasing interpulse interval; Wieland (1960) later obtained similar results. Solomonow, Raplee, and Lyman (1978) found two-point thresholds to depend on pulse frequency, time delay, and pulse width, the last two variables interacting. More significantly, Solomonow, Lyman, and Freedy (1977) uncovered 
evidence of anisotropy in that two-point thresholds varied with body site, being smaller on the limbs than on the trunk. Gilmer (1961) reported evidence of anisotropy with respect to orientation of electrodes; without specifying details, he noted that "the sense of 'twoness' appears to be present on the order of mere millimeters of separation as we go around the arm, but a matter of inches up and down the arm" (p.216).

The present study asked subjects to judge the perceived distance between two electrodes pulsed sequentially with brief biphasic stimuli; of particular concern were answers to four questions: (1) How does perceived distance depend on body site? (2) How does perceived distance depend on spatial orientation in a given site? (3) How much does perceived distance differ across individual subjects? (4) How accurate and how reliable are the judgments made by individual subjects?

\section{METHOD AND PROCEDURE}

\section{Apparatus and Stimull}

Apparatus, stimuli, and some of the methods were like those already described (Girvin et al., 1982). The stimuli were biphasic $(+/-)$ pulses of constant current, $.5 \times .5 \mathrm{msec}$ in duration, presented, in the main experiment, through the unifoeal electrodes, and, in the secondary experiment, through the concentric electrodes.

\section{Percelved DLatance as a Function of Locus and Orientation}

First, absolute thresholds were measured for each of the 16 electrodes in each of the four arrays, using the procedure of the previous study (Girvin et al., 1982). For the main part of the present study - distance estimation-the levels of current were set at 2.5 times threshold to each electrode (a value that reliably produces detectable and comfortable sensations), with the restriction that the maximal current available was $8 \mathrm{~mA}$.

In each experimental session, four electrode arrays were placed on four of five possible locations/orientations: (1) forehead, horizontal orientation, centered on the midline; (2) forearm, transverse orientation, centered about $2-5 \mathrm{~cm}$ below the elbow; (3) forearm, longitudinal orientation, centered on the volar surface about halfway between wrist and elbow; (4) abdomen, horizontal orientation, just below the rib cage and to the left of the midline; and (5) abdomen, vertical orientation, to the right of the midline.

Each subject served in five sessions, with a different one of the five loci/orientations omitted from each session; thus, each locus/orientation appeared in four of the five sessions for each subject. The electrode arrays were themselves rotated over loci/ orientations for each subject to eliminate any systematic effect that could possibly have arisen from differences amons electrode arrays. Six men served as subjects. Three were among the authors, and therefore familiar with the parameters of stimulation. The other three were partly naive in this respect. Although the subjects did have some knowledse of the largest distances possible, electrode arrays were covered during the sessions. No systematic difference appeared between the results of the informed and relatively uninformed subjects.

After the thresholds were determined, the computer calculated all of the possible pairs of electrodes in each array for which thresholds could be determined and which produced the physical distances of $0,5,10,20,40,70,100$, and $140 \mathrm{~mm}$. The computer then generated a random sequence over which the stimulus pairs would be presented. Loci/orientations and distances were randomized. Sometimes, especially with the greatest distances, only one or two different electrode pairs could produce a given distance; more often, several pairs could. Selections were made randomly from all the electrode pairs that could give each distance. Each distance in each array was presented for judgment a total of 10 times in a session, making an overall total of 4 loci $\times 7$ distances $\times 10$ judgments $=280$ stimulations per session.

An audible warning came $1.5 \mathrm{sec}$ before the first stimulus of each pair; the warning was coded to inform the subject of the location of the upcoming stimuli. The stimuli in each pair were $.5 \times .5 \mathrm{msec}$ in duration, separated in time by an onset asynchrony of $.24 \mathrm{sec}$ and accompanied by simultaneous audible signals that were discriminable from the warning signal. The subject responded by estimating the distance between stimuli in millimeters; he did this by entering a three-digit number on the touchtone keyboard. A time interval of $8.5 \mathrm{sec}$ was allotted between the presentation of each stimulus pair and the warning signal to the next pair; if a three-digit number was not entered in that period, no response was recorded, and the stimulus pair was repeated once at the end of the session. If the first, the second, or both stimuli in each pair were not perceived, the subject could so indicate through the touch-tone, and again the stimulus pair would be repeated once at the end of the session. Pauses for rest occurred after each set of 120 stimulus pairs.

\section{Effeet of Onset Aaynchrony}

In order to determine to what extent the results of the main experiment might depend on the particular time interval $(.24 \mathrm{sec})$ between members of each stimulus pair, a second experiment was conducted. Here, only two loci/orientations (forehead, horizontal; abdomen, horizontal) and four physical distances (40, $70,100,140 \mathrm{~mm}$ ) were employed. This experiment was conducted using the concentric electrodes provided by Saunders. Extensive preliminary experiments showed no difference between the two types of electrodes with respect to perceived distance. Through the concentric electrodes, however, perhaps because of their greater surface area, stimulation more reliably aroused threshold and suprathreshold sensations.

Two of the subjects from the main experiment each served in two sessions, one for each body locus, in which stimuli at each of the four distances were presented for judgment 10 times at each of seven onset asynchronies $(.002, .04, .07, .13, .24$, .48 , and $.96 \mathrm{sec}$ ), again making 280 stimulus pairs per session.

\section{Visual Dhance Eutimation}

To control in part for any individual differences in perception or conception of distance in millimeters, each of the subjects who served in the main experiment also participated in a study of visual distance estimation. Dot stimuli, presented in the same temporal sequence as the electrocutaneous stimuli in the main experiment, were programmed to appear on the visual monitor of the computer. For every subject, 14 distances between dots (range $=0$ to $140 \mathrm{~mm}$ ) were presented in random order for estimation. Bach visual extent was presented and judged by each subject five times in the single session; the 14 stimulus distances were divided into two groups of 7 (each covering essentially the full range of distances), every session thereby being divided into two subsessions containing 35 stimulations each $(7$ distances $\times 5$ judgments). The subjects viewed the screen with both eyes from a distance of about $1 \mathrm{~m}$ in a weakly illuminated room.

\section{RESULTS AND DISCUSSION}

\section{Data Averaging}

The individual distance estimations given to stimuli at each locus, orientation, and separation were both averaged within subjects and pooled across sub- 
jects. Although the experiment was designed so that each physical distance could be judged a total of 40 times by each subject over all sessions, in several instances smaller numbers of judgments were obtained. Occasionally, a stimulus pair did not evoke a numerical response, generally because one or both members of the pair were not perceived on a given trial. Often thresholds could not be measured on one or more electrodes. Although the electrode arrays were designed such that many of the physical distances could be produced by several different pairs of electrodes, not all distances could be generated by multiple pairs. In particular, the largest stimulus distance $(140 \mathrm{~mm})$ usually could be formed only by a single pair of electrodes. Consequently, if a threshold could not be measured in a given session on either of those electrodes, it was not possible to provide that longest distance. Indeed, when the largest distance $(140 \mathrm{~mm})$ was presented vertically to the abdomen, three of the six subjects were unable to give judgments in any of the four sessions in which that stimulus could appear. (Furthermore, mechanical failures over time in some of the electrode arrays precluded the full use of some of the electrodes.) Because the number of judgments per stimulus varied across stimuli and across subjects, the results, shown in Figure 1, represent the outcome of averaging for each subject and then pooling the subjects' averages-rather than pooling all the raw data.

\section{Percelved Distance as a Function of Body \\ Locus and Orientation: Anisotropy}

The major feature apparent both in the individual functions and in the pooled functions is the skin's anisotropy. Except at very small physical distances,

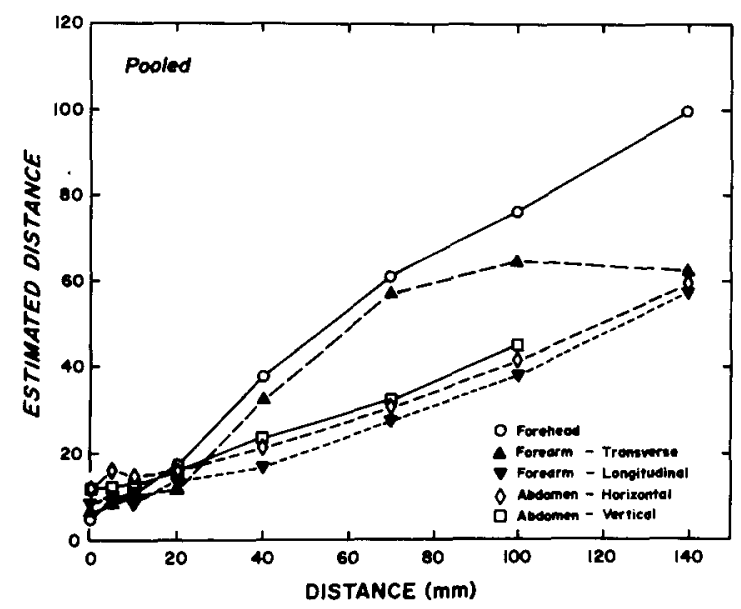

Figure 1. Dotimated distance as a function of setnal distance In millimeters between electrocutaneous stimul for different body loci and orkentation, pooled over the aix subjects. presentation of stimuli to the forehead as compared with any other locus yielded greater perceived distances in terms of the relative size of the numbers; moreover, the forehead produced the most nearly "veridical" judgments. The abdomen, in both of the orientations used, was uniformly poorest, that is, gave the smallest distance estimations. Data for the forearm fell between, with a clear difference between transverse and longitudinal orientations. Over much of the stimulus range, the transverse orientation on the forearm keeps up with the forehead, but at the longest physical distances, the pooled judgments actually decline. This decline is seen in most but not all of the individual functions (Figures 2-7). The decline may well reflect the ambiguity of the direction of distance between two distant points around the forearm, as the points come back toward one another from "behind."

\section{Judgments of Short and Long Distances}

Another prominent feature of the results is the difference between responses to short $(<20-\mathrm{mm})$ and long $(>20-\mathrm{mm})$ distances. Below $20 \mathrm{~mm}$, there is little discrimination among the different physical distances; it is only when the physical separation between electrodes exceeds $20 \mathrm{~mm}$ that the judgments increase steadily and with clear slope. The difference between small and long distances is most apparent when the pooled data are viewed in a logarithmic plot-see Figure 8. Presumably, the short distances fall in the region in which two-point electrocutaneous discrimination is itself probabilistic. Note that Saunders (1974) reported "dynamic" two-point thresholds (thresholds with a stimulus onset asynchrony of $.25 \mathrm{sec}$, about the same value used here) of $6-9 \mathrm{~mm}$ on the abdomen.

A curious fact is that, in the logarithmic plot of Figure 8, anisotropy seems to reverse itself at short distances. Below $20 \mathrm{~mm}$, the forehead gives the smallest distance judgments. What actually is happening is that at distance $=0$, the judgments are reflecting the variability in spatial localization. Consider two stimuli that are presented in succession to the identical spot on the skin. The worse the spatial acuity is and the poorer the ability to localize at that site, the more likely it is that the two stimulations will seem to arrive at different points-and hence the greater will be the average distance estimation.

On the forehead, where acuity is the keenest, spatial noisiness is least and judgments are smallest at a physical separation of zero; on the abdomen, in contrast, where acuity is poor and spatial noise is high, the judgments are greatest; on the forearm, both acuity and distance judgments fall in between. It is only when the actual physical separations increase beyond $20 \mathrm{~mm}$, to values at which the noise 

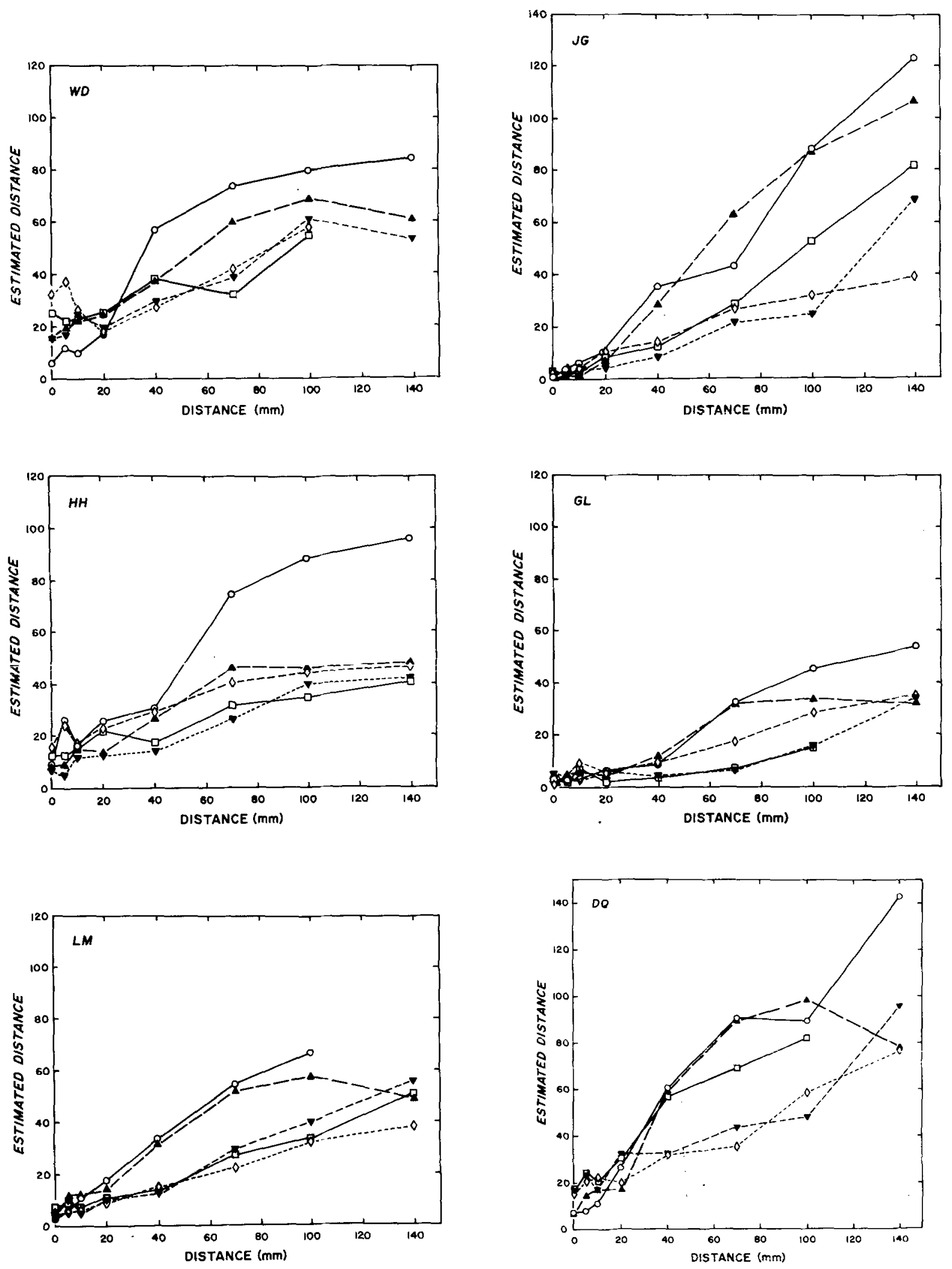

Figures 2-7. As in Figure 1, for individual subjects. 


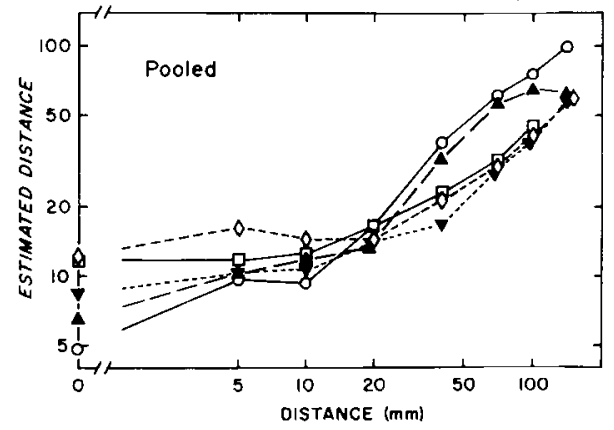

Figure 8. The data of Figure 1 plotted in logarithmic axes.

becomes small relative to the stimulus distances, that distance judgments become reliable, and that the skin's intrinsic anisotropy reveals itself directly in the ordering of the estimations.

\section{Individual Differences}

The psychophysical functions for individual subjects, displayed in Figures 2-7, exhibit considerable overall similarity, although they display a few notable differences as well. As already mentioned, the anisotropy evident in the pooled data also reveals itself in individual functions, with the forehead yielding greatest perceived distances and, in general, the abdomen (both in the vertical and horizontal orientations) yielding smallest distances. Some individual differences emerge, however, in that, for example, J.G. and D.Q., as compared with the other subjects, show relatively high "sensitivity" to distance on the horizontal abdomen. Note, too, that the psychophysical function for the transverse forearm turns over at the longest distance in some individuals, notably L.M., W.D., D.Q., and G.L., but not in J.G.

Although the individual differences in anisotropy are interesting, the single most striking difference evident in the sets of individual functions relates to the size of the scale unit. Recall that the instructions to the subjects were to try to estimate the distances in millimeters. While D.Q.'s judgments are, at least on the forehead, nearly veridical, all other subjects' judgments appear to display "compression," in that the numerical responses fail to keep up with the actual stimulus distances. In some subjects, this effect is dramatic. G.L., for instance, even on the forehead, gave numbers that were typically smaller than half the size of the actual physical values. On the whole, D.Q. and J.G. gave the largest numbers, producing results closest to veridicality, while G.L. and L.M. gave the smallest ones.

Wherein resides the basis for this difference across individuals? Is the difference perceptual, or perhaps judgmental? One possibility is that the differences in scale merely reflect differences in the subjects' conceptions of a millimeter's distance. In order to assess this possibility, we conducted the visual estimation experiment (see Figure 9). The results of that study show that all of the subjects are reasonably able to give veridical judgments of visual length in millimeters, and thus suggest that the subjects' conceptions of unit length are not responsible for the idiosyncratic scale units found in the main experiment. The "compression" in the judgments of the electrocutaneous distance seems to be real, analogous to Green's (1982) findings of compression in distance estimations of mechanical stimuli delivered to the skin.

The visual experiment does not totally resolve the issue, in part because there is not universal agreement as to how cross-modal judgments of space are made. One possibility is that the senses can perceive and provide independent and more or less equivalent information (Marks, 1978), in which case the individual differences reported here are likely to reflect differences among subjects in their tactual distance sensations. Another possibility is that tactual spatial relations are explicitly or implicitly translated into another spatial reference system-probably into visual space (Attneave \& Benson, 1969); in this case, the individual differences could represent either differences in the skin's responses to spatial distance or differences in the subjects' abilities to transfer the information without loss or distortion.

\section{Variability}

Some comments are in order with regard to the variability in the electrocutaneous distance judgments. As compared with the variability seen in the visual distance estimates, which ran on the average

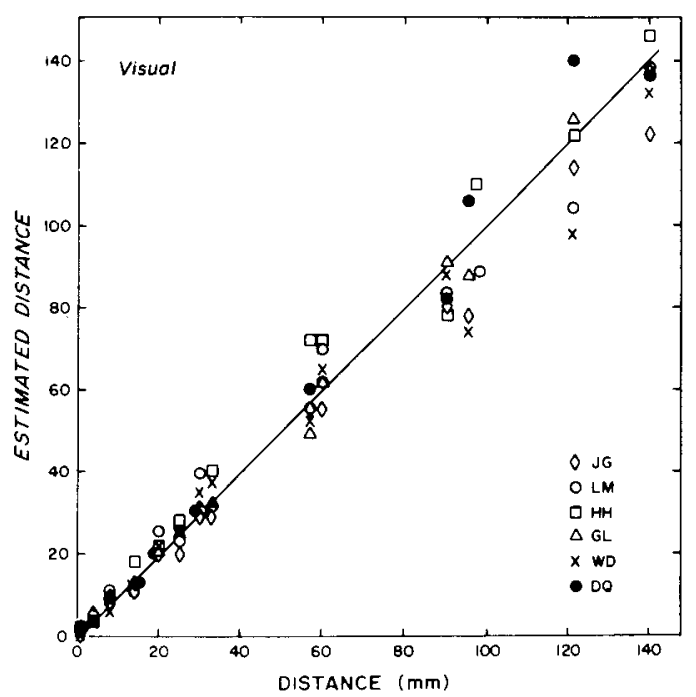

Figare 9. Estimated distance as a function of actual distance in millimeters between viaually presented stimull. 
$8 \%-10 \%$ of the means, the variability in electrocutaneous distance estimates ran from about $15 \%$ to $75 \%$, values two and more times as great. There are undoubtedly several sources for the greater variability in the responses to electrocutaneous stimulation. For one, the visual stimuli were viewed on the computer's video monitor, which itself provided a constant spatial framework against which the judgments could be made. With electrical stimulation, there were no comparable tactual reference points. Second, the visual sense is much more precise than the skin is with respect to spatial localization and acuity. Third, to the extent that judgments of tactual distance may require the information about distance to be translated into a visual representation, it is likely that the very process of translation will add variability. Finally, the electrocutaneous sensations varied somewhat in quality and intensity, and such variations in the individual sensations may have contributed to variability in perceived distance.

One interesting feature of the variability is the way it depends on distance. The top graph in Figure 10 shows standard deviations (pooled across subjects) plotted as a function of perceived distance. By plotting each standard deviation against the perceived distance, data for different distances and loci/orientations tend to collapse onto a single function: It is more the perceived distance (Figure 10, top) than the actual distance (Figure 10, bottom) or location that determines the degree of variability. Over the shorter distances, the standard deviation increases as perceived distance increases, but eventually the standard deviation tends to level off at a value around 17. At the high end of the function, this means a ratio or standard deviation/mean of about $17 \%$; at the low end, the standard deviation can be as much as $50 \%$, or even $75 \%$, of the mean. Although these ratios are large as compared with the variability in spatial judgments made by the visual sense, the variability in electrocutaneous distance estimation does lie in a range comparable to that found in experiments in which subjects give magnitude estimates of perceived intensity. Reanalysis of the data of Marks and Cain (1972) on perceived weight and perceived roughness, for instance, shows ratios of the standard deviation/mean ranging from about $10 \%$ to $20 \%$ (Marks, 1982). For loudness, Green and Luce (1974) reported ratios of $10 \%$ $30 \%$; for brightness, reanalysis of data of Marks (1971; 1-deg field, foveal stimulus) yields a value of $20 \%$. To a first-order approximation, the skin's relative spatial response to electrical stimulation appears about as noisy as the skin's, the ear's, or the eye's response to gradations in mechanical or photic energy.

One final point on variability: Individual differences appear here, too, in that some of the subjects
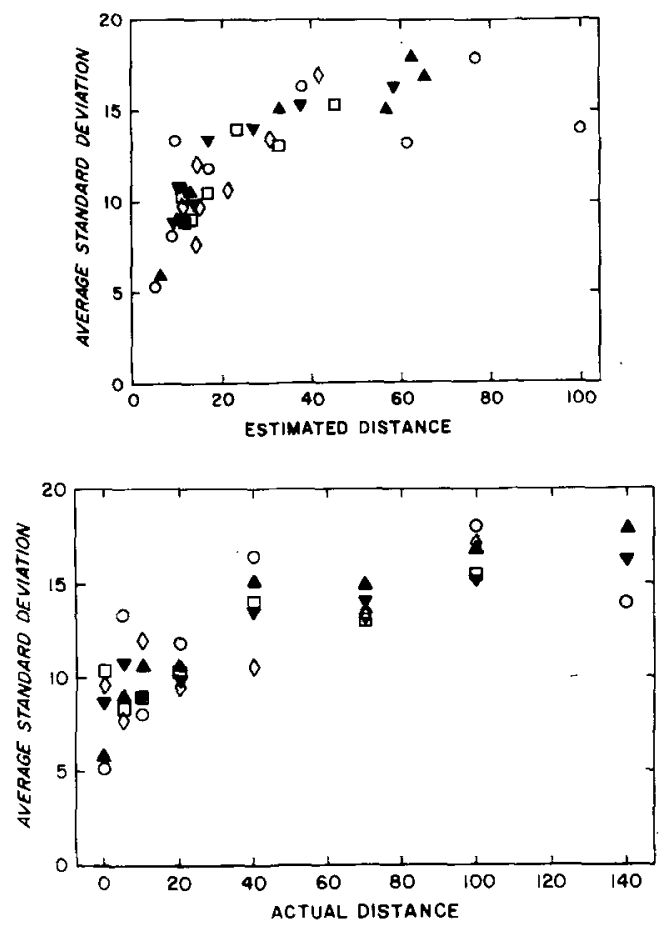

Figure 10. Standard deviations of the unmerical diatance eatimates of electrocutaneous atimull plotied a function of the mean diatance extimates (top) and as a function of the actual distances (bottom).

(e.g., L.M.) gave relatively small ratios of standard deviation to mean, while others (notably G.L.) gave relatively high ratios. Although it is possible that the differences in variability result from differences in underlying spatial acuity-and presumably in the spatial information available through the neural traffic evoked by stimulation at two points-it is more reasonable to assume at this point that these differences derive from differences in such factors as attention and constancy of the conceptual scale unit, that is, in factors involved in the process of judgment per se.

\section{Percelved Distance Related \\ to Temporal Parameters}

To what extent might the outcome of the main experiment-in particular, the marked degree of "compression"-be the result of the .24-sec asynchrony between the onsets of the two pulses that formed each stimulus? That temporal factors can interact with spatial ones in determining perception is well known. In the so-called "tau effect," the temporal spacing between stimuli modifies their perceived spatial separation; if three stimuli, A, B, and $C$, are lined up in a row, with the distance $\mathbf{A B}$ equal to $B C$, and these are activated in sequence, with $A$ followed quickly by $B$ and $B$ less quickly by 
C, then A and B will appear closer together in space than B and C (Helson \& King, 1931). The "rabbit phenomenon" of Geldard and Sherrick (1972), first investigated with vibrotactile stimulation but later extended to other domains, including the electrocutaneous (Geldard, 1975), is perhaps related: With short time intervals between mechanical taps or electrical pulses, the perceived location of the second of two stimuli can be drawn toward the location of the first. (Given a series of pulses to one spot followed at an appropriate time interval by a series of pulses to another spot, a person perceives something like a rabbit hopping down intermediate points along the skin.) In a nutshell, perceived spatial separations can be modified by shifting the time intervals between stimuli.

Mutual attractions like those found in the rabbit phenomenon could produce "compression." Geldard's results (1975, p. 65) show the electrocutaneous as well as the mechanical rabbit to take place over interpulse intervals of about $.02-.275 \mathrm{sec}$, a temporal region that contains the stimulus onset asynchrony used in the main experiment here. Moreover, the perception of distance between mechanical pulses depends strongly on temporal asynchrony (Cholewiak, Note 1). Thus, it is possible that some of the compression found here resulted from spatialtemporal interactions between the sensory effects of the two pulses.

Figures 11 and 12 give the results of the secondary experiment, in which two subjects judged each of four distances at each of seven stimulus onset asynchronies (SOAs). The figures make it clear that the temporal relationship within each pair did have some systematic effect on perceived distance, with the apparent distance between pulses greater with the longest time intervals $(.48$ and $.96 \mathrm{sec})$. Shorter intervals produced greater "compression," although the differences are small as SOA decreases

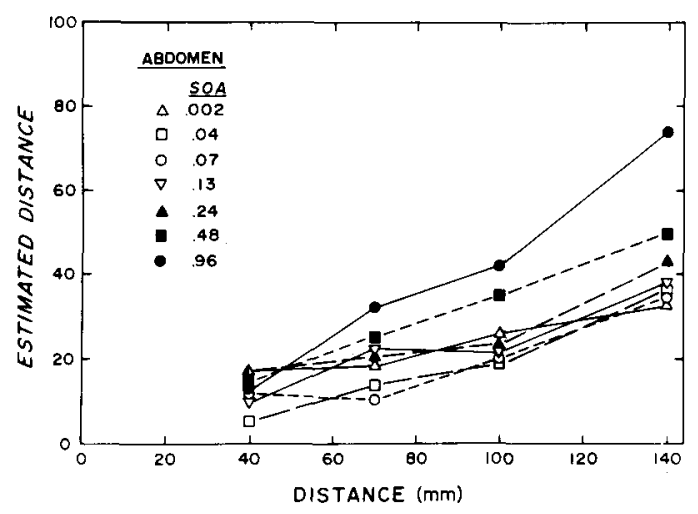

Figure 11. Eutimated distance as a function of actual distance In millimeters between electrocutaneous stimull at varlous temporal asynchronies (SOAs), for the forehead.

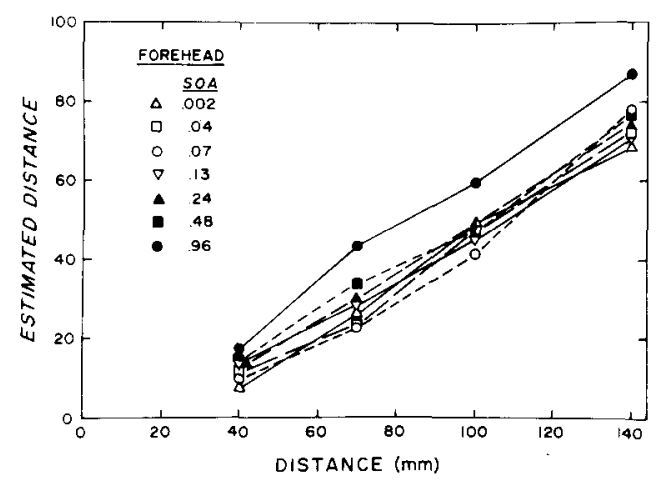

Figure 12. As in Figure 11, for the abdomen.

from .24 to $.002 \mathrm{sec}$. The effects appear smaller than those of the electrocutaneous "rabbit" (Oeldard, 1975), perhaps stemming from the present subjects' knowledge of the maximal possible distances. At no value of SOA, for either the forehead or the abdomen, do the distance judgments achieve veridicality. Compression seems to be the rule, although to a greater or lesser extent, depending on the temporal proximity of the electrical pulses.

\section{GENERAL DISCUSSION}

The major findings are clear. First, perceived distance between electrically stimulated points on the skin is in some sense nonveridical as compared with the actual distance, certainly "compressed" as compared with visually perceived distance. Second, the skin is anisotropic-perceived distance depends on the body site and orientation of the pair of electrodes. The interpretation of these results depends on whether electrical stimulation, like mechanical stimulation, operates by directly arousing peripheral receptors. By way of contrast, electrical stimulation may operate largely by directly arousing en passage cutaneous nerve fibers.

That the skin is anisotropic in its response to mechanical stimulation was suggested over a century ago by Weber (1834) and has been demonstrated recently in a series of experiments by Oreen (1982). That electrical and mechanical stimulation show similarity in this respect implies that both kinds of stimuli may tap the same or related spatial discriminative mechanisms in the somesthetic system. As Oreen suggested, the differences in perceived distance may reflect the operation of differently shaped and sized receptive fields subserving various body regions. Anisotropy and differences in perceived distance imply that different regions of the skin organize space differently. Rather than being learned, such spatial differences are presumably built into the skin's space perception. Indeed, they seem not even to be unlearned: one might expect that, with experi- 
ence, the same distance would always appear the same, but, apparently, this is not the case (cf. Marks, 1978). Note, however, that this explanation can apply fully to electrical stimulation only if such stimulation arouses cutaneous receptors directly.

Rollman (1974) has presented evidence that electrical stimulation excites nerve fibers directly. Such a mode of stimulation requires a different kind of explanation for the present results. For example, two points stimulated along the same nerve bundle would be perceived as closer together than two points stimulated on different nerve bundles. Given the neurounatomical innervation of the skin, this would produce relative compression along the horizontal axis of a limb.

As indicated in the introduction, this study was motivated by the desire to develop devices that transmit information by electrical stimulation of the skin, particularly an electrocutaneous speech aid for the deaf. To that end, the findings here might be taken to imply that, to the extent that spatial discriminations may be necessary or desirable-and specifically, to the extent that relatively veridical spatial representations may be desirable-the forehead is preferable to the forearm or abdomen as a location.

However, several of the subjects noted that the suprathreshold sensations on the forehead often had a distinctly unpleasant quality-sharp, burning, stinging, and often spatially extended. In comparison, stimulation of the abdomen resulted in generally more pleasant, spatially small, tap-like qualities, no small consideration for a prosthetic device.

It should be noted, however, that, while the forehead gave the greatest range of spatial responsespsychological unit for physical unit-the absolute size of the typical person's forehead is considerably smaller than that of his or her abdomen. Thus, it may be quite easy to achieve perceived distances on the abdomen as great as or greater than those on the forehead, simply by using much greater physical separations between stimuli. It is significant in this respect that the variability in spatial response seems to depend more on the perceived distance than on the actual physical distance or body locus. Thus, there may be no intrinsic disadvantage-from the point of view of perceived distance-to the use of the abdomen as a site for an electrocutaneous speech aid.

\section{RDPRENCE NOTE}

1. Cholewiak, R. W. Spatiotemporal integration in the perception of tactlle distance. Paper presented at 22nd Annual Meeting of the Psychonomic Society, Philadelphia, November 1981.

\section{REPTRENCES}

Atrneave, F., \& Benson, B. Spatial coding of tactual information. Journal of Experimental Psychology, 1969, 81, 216-222.

Geldond, F. A. Sensory saltation: Metastability in the perceptual world. Hillsdale, N.J: Erlbaum, 1975.

Geldord, F. A., \& Sherricx, C. E., Jn. The cutaneous "rabbit"; a perceptual illusion. Science, 1972, 178, 178-179.

Gilmer, B, von H. Toward cutaneous electropulse communication. Journal of Psychology, 1961, 52, 211-222.

Gravin, J. P., Marks, L. E., Antunes, J. L., Quest, D. O., O'Keere, M. D., Ning, P., \& Dobelle, W. H. Electrocutaneous stimulation $I$. The effects of stimulus parameters on absolute threshold. Perception \& Psychophysics, 1982, 32, 524-528.

Grean, B. G. The perception of distance and location for dual tactile pressures. Perception \& Psychophysics, 1982, 31, 315-323.

GresN, D. M., Luce, R. D. Variability of magnitude estimates: A timing theory analysis. Perception \& Psychophysics, 1974, 15, 291-300.

Herson, H., \& Kina, S. M. The tau effect: An example of psychological relativity. Journal of Experimental Psychology, $1931,14,202-217$.

JoNE8, F. N. Space-time relationships in somesthetic localization. Science, 1956, $124,484$.

Marks, L. E. Brightness and retinal locus: Effects of target size and spectral composition. Perception \& Psychophysics, 1971, 9, 26-30.

MARxs, L. E. The unity of the senses: Interrelations among the modalities. New York: Academic Press, 1978.

Marks, L. E. Psychophysical measurement: Procedures, tasks, scales. In B. Wegener (Bd.), Social attlitudes and psychophysical measurement. Hillsdale, N.J: Erlbaum, 1982.

Marks, L. E., \& Cain, W. S. Perception of intervals and magnitudes for three prothetic continua. Journal of Experimental Psychology, 1972, 94, 6-17.

Rouryax, G. B. Electrocutaneous stimulation. In F. A. Geldard (Ed.), Cutaneous communication systems and devices. Austin, Tex: Psychonomic Society, 1974.

Saunders, F. A. Electrocutaneous displays. In F. A. Geldard (Bd.), Cutaneous communication systems and devices. Austin, Tex: Psychonomic Society, 1974.

Solomonow, M., Lyman, J., \& Fresdy, A. Electrotactile twopoint discrimination as a function of frequency, body site, laterality, and stimulation codes. Annals of Biomedical Engineering, 1977, 5, 47-60.

Solomonow, M., Raplez, L., \& Lyman, J. Electrotactile twopoint discrimination as a function of frequency, pulse width and pulse time delay. Annals of Biomedical Engineering, $1978,6,117-125$.

Vinzonot, K. Ueber die Ursachen der verschiedenen Entwickelung des Ortsinnes der Haut. Archiv fur die Gesamte Physiologie, 1869, 2, 297-306.

Wresn, E. H. De pulsu, resorptione, auditu et tactu: Annotationes anatomicae et physiologicae. Leipzig: Kohler, 1834.

Weinerein, S. Intensive and extensive aspects of tactile sensitivity as a function of body part, sex, and laterality. In D. R. Kenshalo (Ed.), The skin senses. Springfield, Ill: Thomas, 1968.

WIELAND, B. A. The interaction of space and time in cutaneous perception. American Journal of Psychology, 1960, 73, 248-255.

(Manuscript received January 4, 1982;

revision accepted for publication September 9, 1982.) 